\title{
A dual-loop tracking control approach to precise nanopositioning
}

\author{
Mohammed Altaher, Douglas Russell and Sumeet S. Aphale* \\ School of Engineering, University of Aberdeen, UK
}

Abstract

Nanopositioners are mechanical devices that can accurately move with a resolution in the nanometre scale. Due to their mechanical construction and the piezoelectric actuators popularly employed in nanopositioners, these devices have severe performance limitations due to resonance, hysteresis and creep. A number of techniques to control nanopositioners, both in open-loop and closed-loop, have been reported in literature. Closed-loop techniques clearly outperform open-loop techniques due to several desirable characteristics, such as robustness, high-bandwidth, absence of the need for tuning and high stability along with others. The most popular closed-loop control technique reported is one where a damping controller is first employed in an inner loop to damp the mechanical resonance of the nanopositioner, thereby increasing achievable bandwidth. Consequently, a tracking controller, typically an Integral controller or a Proportional-Integral controller, is implemented in the outer loop to enforce tracking of the reference signal, thereby reducing the positioning errors due to hysteresis and creep dynamics of the employed actuator. The most popular trajectory a nanopositioner is forced to track is a raster scan, which is generated by making one axis of the nanopositioner follow a triangular trajectory and the other follow a slow ramp or staircase. It is quite clear that a triangle wave (a finite velocity, zero acceleration signal) cannot be perfectly tracked by a first-order integrator and a double integrator is necessary to deliver error-free tracking. However, due to the phase profile of the damped closed-loop system, implementing a double integrator is difficult. This paper proposes a method by which to implement two integrators focused on the tracking performance. The criteria for gain selection, stability analysis, error analysis, simulations and experimental results are provided. These demonstrate a reduction in positioning error by $50 \%$, when compared to the traditional damping plus first-order integral tracking approach.

Keywords

Nanopositioning, Robustness, Damping and Tracking, Error Analysis

\section{Introduction}

Nanopositioning systems are ideal for applications requiring precise positioning with nanoscale resolution. Consequently, they are employed as positioning stages in Atomic Force Microscopes Binnig et al. (1986), High Density Data Storage Devices Sebastian et al. (2008), lab-on-chip devices Sarella et al. (2014), nanolithography Gao et al. (2017), optical systems Lee (2017) and nanomachining Zhu et al. (2017) etc.

*Corresponding author; email: s.aphale@ abdn.ac.uk 
Nanopositioners are usually capable of motion along $x-y$ or $x-y-z$ axes. For most $-x-y$ nanopositioners, the popular scan trajectory they are expected to follow is a raster, which is generated by making one axis of the nanopositioner trace a triangular trajectory while the other axis traces a slow ramp or staircase Ju et al. (2014). The triangular trajectory is a high-bandwidth signal (ideally composed of infinite harmonics of the fundamental frequency wave) and as a result it has the potential to excite the resonant mode of the particular nanopositioner axis; this is an almost unavoidable artefact of the mechanical design of most nanopositioners Yong et al. (2014). This resonant mode limits the open-loop operational

bandwidth of most nanopositioners to a frequency of about $\frac{1}{100}^{\text {th }}$ of the resonant mode frequency, Fleming \& Wills (2009). The positioning performance is further degraded because of hysteresis Wang et al. (2014) and creep effects Mazlan \& Ripin (2017) arising from the piezoelectric actuators popularly employed in nanopositioners. This is due to their desirable properties, such as high-bandwidth, ease of actuation and system integration, repeatability, nearly atomic-level resolution, frictionless and stictionless motion etc, Gu et al. (2016). To overcome these performance limitations, nanopositioners are seldom used without some sort of control algorithm employed to correct for such adverse effects, Devasia et al. (2007).

The most popular closed-loop control technique employed to improve the positioning performance of nanopositioners is one where two control loops are implemented simultaneously to combine both damping and tracking. The inner loop implements a suitable damping controller to impart substantial damping to the dominant resonant mode. This, in turn facilitates a high-gain tracking controller to be implemented, which minimises the positioning errors introduced by the nonlinear phenomena of hysteresis and creep Rana et al. (2018). For the damping loop, any controller from a choice of Positive Position Feedback (PPF) Fanson \& Caughey (1990), Positive Velocity and Position Feedback (PVPF) Bhikkaji et al. (2007), Resonant Control Pota et al. (2002), Shunt Control Hagood \& von Flotow (1991), Integral Resonant Control (IRC) Teo et al. (2014) and Robust Control Sebastian \& Salapaka (2005) is employed. Tracking is usually enforced by using an Integral (I) Orszulik \& Shan (2017), or a Proportional-Integral (PI) Li et al. (2017), controller. Proportional-Integral-Derivative (PID) control is seldom used, Fleming \& Leang (2014). Over the years, design improvements in each of these controllers have been reported specifically for nanopositioning applications, Soto \& Adeli (2017), San-Millan et al. (2017). Until recently, the design of the damping controller and the tracking controller was performed in a sequential fashion: damping first tracking later. Recent work, however, has shown that simultaneous design of the damping and tracking control schemes delivers a substantially superior positioning performance over a wider bandwidth, Namavar et al. (2014), Russell et al. (2016). Yet, all these advances still employ a first-order integrator to track a triangular trajectory: a trajectory with a finite, non-zero velocity. Therefore, achieving zero-error positioning (excluding noise effects) is not yet possible. It is well-known that to track a finite, non-zero velocity trajectory with zero steady-state error, a double-integrator is needed, Barrett \& Quate (1991). However, implementing a double-integral tracking controller is difficult due to the phase profile of the closed-loop damped system.

Of the damping controllers listed above, the IRC design results in the lowest-order damping controller (typically $1^{s t}$-order). This paper proposes a method to integrate a suitably designed IRC damping controller with a second integrator through a dual-loop tracking control strategy. After a brief description of the experimental system and an overview of the system model in Section 2, the proposed control scheme will be described in detail in Section 3. The experimental results in time-domain and frequency-domain are reported in Section 4. Error and robustness analysis is also included. The paper ends with concluding remarks in Section 5.

\section{System Identification and Modelling}

This section is divided into two subsections. Subsection 2.1 will describe the experimental test system utilised to validate the proposed control scheme. Subsection 2.2 will briefly describe the frequency-domain system identification performed and the frequency-domain model derived that accurately captures the dynamics of one axis of the nanopositioner within the bandwidth of interest. This model will later be used in the control design and initial analysis. 


\subsection{Experimental Setup}

Figure 1 (a) presents a snapshot of the experimental setup while (b) is a schematic drawing to illustrate the clear communication of each element of the nanopositioner. It consists of a two-axes $(x-y)$ serial kinematic piezoelectric-stack driven flexure-based nanopositioner. Each axis is driven by a $10 \mathrm{~mm} 200 \mathrm{~V}$ piezoelectric stack actuator capable of generating $40 \mu \mathrm{m}$ motion along each axis. The nanopositioner also provides integrated mounts for capacitive sensor probes. The MicroSense 6810 capacitive displacement sensor and 6504-01 probe with a sensitivity of $5 \mu \mathrm{m} / \mathrm{V}$ provide a voltage signal proportional to the displacement sensed along each axis. The piezoelectric stack actuators are driven by two PiezoDrive PDL200 voltage amplifiers with a gain of 20. All the open-loop and closed-loop experiments and consequent time-domain data is captured using NI LabVIEW's Real-Time module at a sampling frequency of $20 \mathrm{kHz}$. The frequency response data is recorded using a HP 35670A FFT Dynamic Signal Analyzer (DSA).

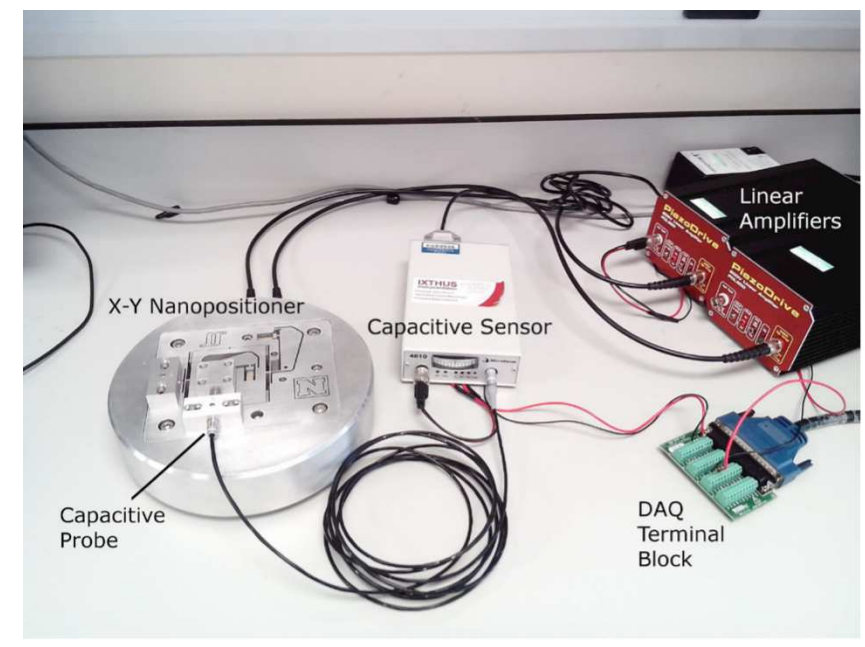

(a)

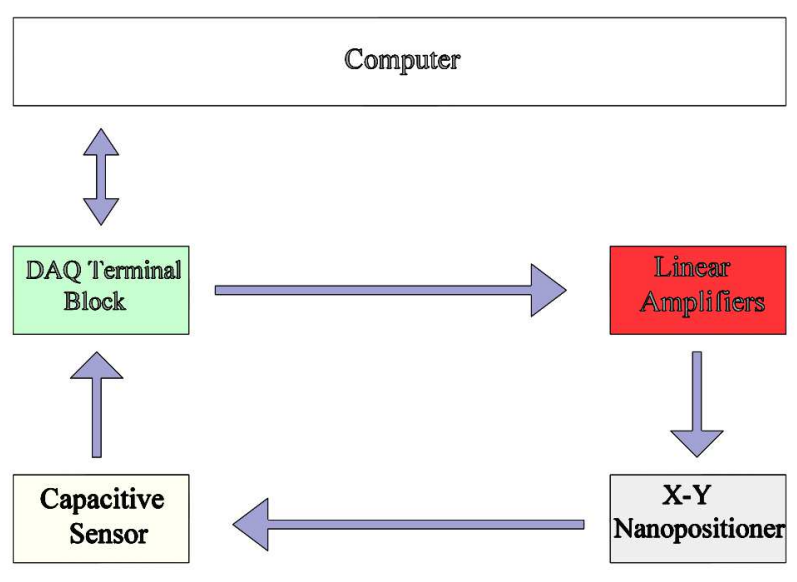

(b)

Figure 1. (a) A two axis serial-kinematic nanopositioning platform with a range of $40 \mu \mathrm{m}$ driven by two PiezoDrive $200 \mathrm{~V}$ linear voltage amplifiers, with position measured by capacitive sensor; (b) A schematic drawing showing the experimental setup of the nanopositioner components.

\subsection{System Identification and Modelling}

The open-loop frequency response of one axis of the nanopositioner is obtained by sending a band-limited random noise input generated by the DSA of amplitude $0.25 V_{p k}$ within the frequency range $20 \mathrm{~Hz}-2 \mathrm{kHz}$. This signal was fed to the voltage amplifier as input and the output of the amplifier was used to excite the piezoelectric stack. This input corresponds to a displacement within 0-2 $\mu \mathrm{m}$, or $5 \%$ of the total range of the platform. Throughout this work, the piezoelectric stack actuator was kept at a bias of $+100 \mathrm{~V}$ to avoid depolarisation due to the application of negative voltages. As the identification was done from $20 \mathrm{~Hz}$ onwards, the effect of creep was eliminated due to the absence of any low-frequency components. The piezoelectric stack actuator for the other axis was shorted to eliminate its cross-coupling effect. The recorded frequency response (magnitude only) is shown in Figure 2. From this frequency response, the following conclusions can be drawn:

- The DC gain of the entire open-loop system (voltage amplifier - piezoelectric stack actuator - mechanical axis of the platform - capacitive displacement sensor) is $-6.2 \mathrm{~dB}$.

- The dominant resonant mode of this axis occurs at a frequency of $716 \mathrm{~Hz}$.

- There are a couple of low-magnitude modes seen within the bandwidth of interest, but these are beyond the dominant mode. These can be safely ignored due to their small dynamic range compared to that of the dominant mode at 716 


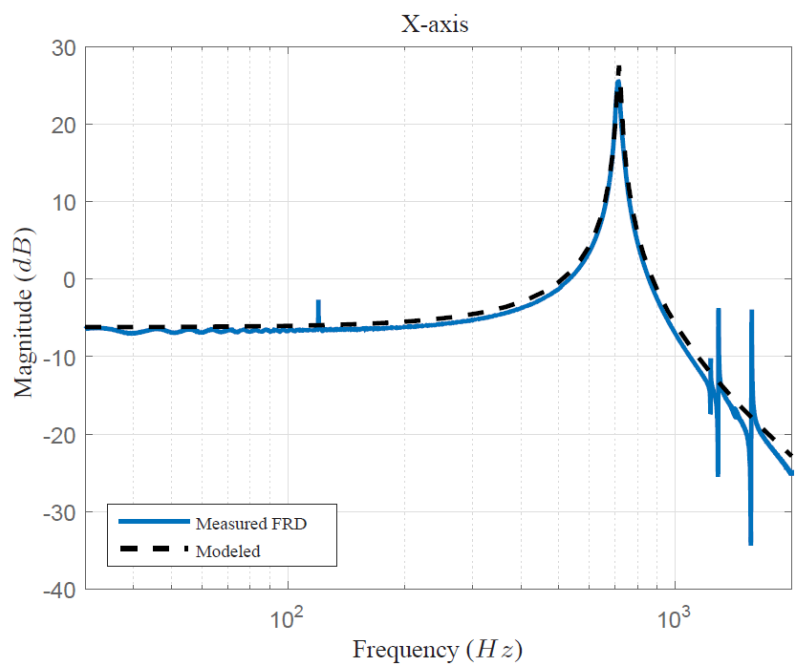

Figure 2. comparison between the measured magnitude experimental response and modelled for the x-axis of the system in the open-loop.

$H z$. Consequently, the measured magnitude response looks similar to that exhibited by a typical second-order system with low damping.

The transfer function of a generic second-order system is given by:

$$
G(s)=\frac{\sigma^{2}}{s^{2}+2 \zeta \omega_{p} s+\omega_{p}^{2}},
$$

where $\zeta$ is the damping coefficient, $\omega$ is the natural frequency and $\sigma^{2}$ is the DC gain constant of the system. Using a subspace-based modelling technique as described in McKelvey et al. (2002), a second-order model of this axis of the nanopositioner is obtained. The resulting transfer function that accurately captures the relevant dynamics of the axis within the recorded bandwidth of interest is given by:

$$
G(s)=\frac{9.911 \times 10^{6}}{s^{2}+92.12 s+2.026 \times 10^{7}}
$$

This transfer function model is used to design the parameters for the IRC damping controller as well as the gains for the two tracking integrators. A detailed control design is presented in the following section.

\section{Control strategy}

Consider the feedback schemes depicted in Figure 3 (a) and (b). As mentioned earlier, this work aims to improve the positioning performance of a typical control strategy that combines both damping and tracking. In Figure 3 (a), the traditional control scheme with the IRC-based inner damping loop and an outer integral tracking loop is shown. It is well known that this control scheme delivers substantial improvement over the open-loop positioning performance of a nanopositioner axis. Yet, as the popular input to the nanopositioner axis is a triangular trajectory, it is clear that a first-order integrator will not be able to achieve zero-error tracking for this input signal.

As mentioned earlier, the closed-loop phase profile of the damped nanopositioner axis does not furnish sufficient phase margin to allow for the implementation of a second-order integrator, a theoretical requirement for obtaining zero-error tracking of triangular trajectories. Therefore, the next best option is to implement a second tracking loop and evaluate 
whether any improvement in positioning performance is achievable. The block diagram depicting this control strategy is shown in Figure 3(b).

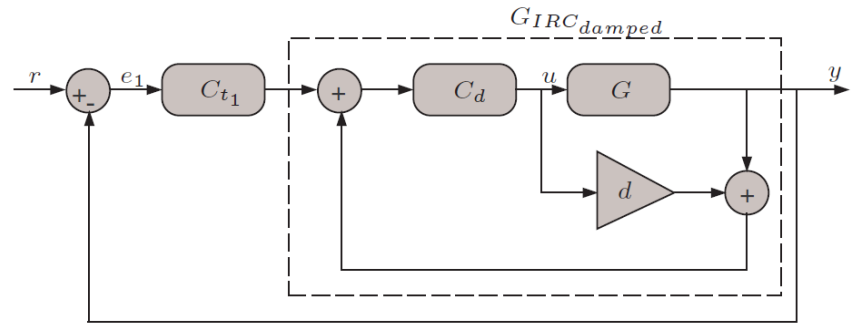

(a)

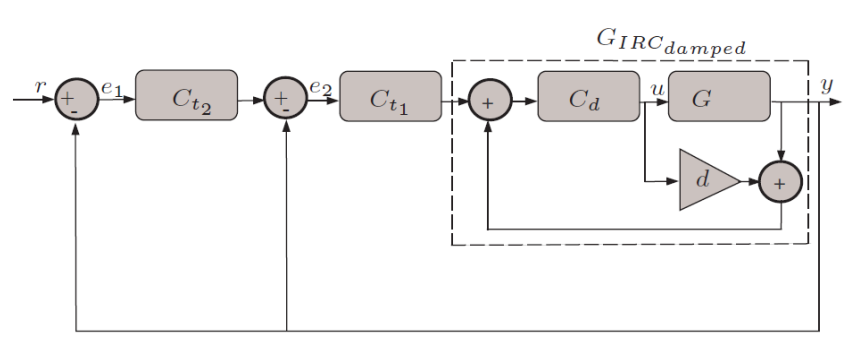

(b)

Figure 3. Block diagrams for (a) the traditional control scheme and (b) the proposed control scheme with dual-loop tracking.

The overall control algorithm in Figure 3 (b) consists of two integral tracking controllers: $C_{t_{1}}$ and $C_{t_{2}}$. The tracking capability of the control algorithm shown in Figure 3 (a) is further augmented by an additional integral tracking controller, $C_{t_{2}}=\frac{K_{t_{2}}(s)}{s}$. Therefore, the overall transfer function for the proposed scheme is:

$$
\frac{Y(s)}{R(s)}=\frac{C_{t_{2}} C_{t_{1}} C_{d} G}{C_{t_{2}} C_{t_{1}} C_{d} G+1-d C_{d}-G C_{d}+C_{t_{1}} C_{d} G}
$$

Where $C_{d}(s)=\frac{K_{d}}{s}$ is the damped system gain, $G$ is the plant and $\mathrm{d}$ is the feed-through term. Thus, the characteristics equation of the proposed dual-loop feedback scheme is given by:

$$
s^{5}+\left(2 \zeta \omega_{p}-d K_{d}\right) s^{4}+\left(\omega_{p}^{2}-2 \zeta \omega_{p} d K_{d}\right) s^{3}+\left(-d K_{d} \omega_{p}^{2}-K_{d} \sigma^{2}\right) s^{2}+K_{t_{1}} K_{d} \sigma^{2} s+K_{t_{1}} K_{t_{2}} K_{d} \sigma^{2}=0
$$

As the value of $\zeta$ is usually small, it can be safely neglected in the overall analysis. Thus, the characteristic equation can be simplified as:

$$
s^{5}-d K_{d} s^{4}+\omega_{p}^{2} s^{3}-\left(d K_{d} \omega_{p}^{2}+K_{d} \sigma^{2}\right) s^{2}+K_{t_{1}} K_{d} \sigma^{2} s+K_{t_{2}} K_{t_{1}} K_{d} \sigma^{2}=0
$$

Overall stability is examined for the proposed control scheme by applying the Routh-Hurwitz stability criterion. The characteristics equation of the proposed scheme is further analysed to determine the range of $K_{t_{1}}, K_{t_{2}}, K_{d}$ and $d$ required for closed-loop stability. For this characteristic polynomial to have all roots with negative real parts, the coefficients of all the ' $s$ ' terms must be positive. Noting that $K_{t_{1}}, K_{t_{2}}$ and $K_{d}$ are positive results in the following inequality to be a necessary condition for stability:

$$
-d K_{d} \omega_{p}^{2}-K_{d} \sigma^{2}>0
$$

The terms in (6) can be rearranged to find the adequate feed-through term:

$$
d<-\frac{\sigma^{2}}{\omega_{p}^{2}}
$$

Similarly, the relationships for the damping gain $K_{d}$ and the tracking controller gains $K_{t_{1}}$ and $K_{t_{2}}$ were obtained. They are presented in (8), (10) and (9).

$$
K_{d}<-\frac{\omega_{p}^{2} d+\sigma^{2}+K_{t_{1}} K_{t_{2}} d}{K_{t_{1}} d^{2}}
$$




$$
\begin{aligned}
K_{t_{1}}> & \frac{K_{d} d \omega_{p}^{2}+\sigma^{2} K_{d}+\omega_{p}^{2} K_{t_{2}}}{K_{t_{2}}^{2}-K_{d}^{2} d^{2}-K_{d} d-K_{d} K_{t_{2}} d} \\
K_{t_{2}}< & \frac{K_{t_{1}}-\omega_{p}^{2}+\sqrt{\left(2 K_{t_{1}} \omega_{p}^{2}+4 K_{t_{1}}^{2} \sigma^{2}+K_{t_{1}}^{2}+\omega_{p}^{4}\right)}}{2 K_{t_{1}}}
\end{aligned}
$$

For the nanopositioner used in this work, the resulting parameter values are:

$$
\begin{gathered}
K_{t_{1}}=3300 \\
K_{t_{2}}=617 \\
K_{d}=2750 \\
d=-1.5
\end{gathered}
$$

Traditionally, the value of the feed-through term must be negative to ensure stability, as noted in equation 7 . The traditional approach to select the tracking and damping gain in nanopositioning is to allow sufficient phase margin to account for unmodelled dynamics. The values of the tracking and damping gains were chosen using the trial and error method and obey equations (7, 8,9 and 10). The obtained stability margins as a result of the chosen values are: Phase Margin (PM) of $62.6^{\circ}$ and Gain Margin (GM) of $6.85 d B$. In the following section the error transfer function for the traditional and proposed dual-loop scheme is presented.

\subsection{Error analysis}

In this section, the concept of error ratio is presented. Error ratio is a measure used to quantify the performance of the controller over a range of frequencies, and the following equation can determine it:

$$
E(s)=\frac{1}{1+G(s) H(s)}
$$

For the traditional control scheme, the error transfer function is given by:

$$
E_{\text {traditional }}(s)=\frac{R(s)\left(1-d C_{d}(s)-G(s) C_{d}(s)\right)}{1-d C_{d}(s)-G(s) C_{d}(s)+C_{t_{1}}(s) C_{d}(s) G(s)}
$$

Similarly, the error transfer function for the proposed dual-loop control strategy depicted in Figure 3 (b) is:

$$
E_{\text {proposed }}(s)=\frac{R(s)\left(1-d C_{d}(s)-G(s) C_{d}(s)+C_{t_{1}}(s) C_{d}(s) G(s)\right)}{1-d C_{d}(s)-G(s) C_{d}(s)+C_{t_{1}}(s) C_{d}(s) G(s)+C_{t_{1}}(s) C_{t_{2}}(s) C_{d}(s) G(s)}
$$

Figure 4 shows the measured error dynamics over the bandwidth-of-interest. The aim of studying the error in the frequency domain is to provide an alternative way of viewing the improvement in positioning performance achieved by the proposed dual-loop tracking scheme. Due to various tracking error sources associated with nanopositioning such as phase delay of the system, resonance-induced vibrations, parameter uncertainty and nonlinear effects such as hysteresis, plotting the error ratio is deemed as the best method to quantify the enhanced performance. It is clear that the proposed dual-loop scheme produces significantly less error compared to the traditional single-loop scheme up to the resonant frequency of the nanopositioner axis. This resonant frequency acts as a crossover point when the single-loop control scheme performs marginally better than the dual-loop scheme. The positioning error is less than the single-loop at high frequencies. As 


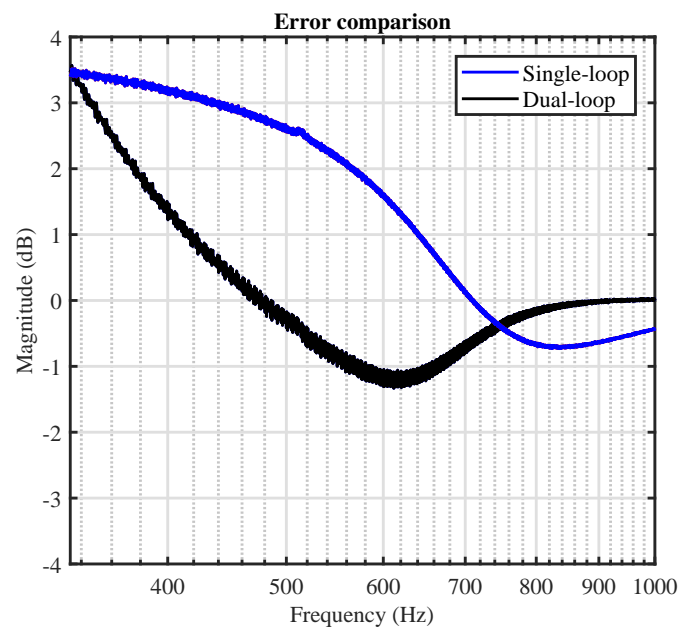

Figure 4. Error dynamics for the traditional single-loop tracking control strategy and the proposed dual-loop tracking control strategy within the bandwidth-of-interest.

the improvement in tracking performance for trajectories within the bandwidth-of-interest outweighs the slightly inferior performance at the higher frequencies that are out-of-band, the proposed dual-loop control strategy is deemed to be a simple yet significant improvement in positioning performance.

The final value theorem is applied to (14):

$$
e_{\text {proposed }}(\infty)=\lim _{s \rightarrow 0} s E_{\text {proposed }}(s)
$$

by substituting $R(s)=\frac{1}{s^{2}}$ in (14) for a ramp-like reference trajectory, which results in:

$$
\begin{aligned}
e_{\text {proposed }_{(\text {steady-state })}}= & \frac{-\sigma^{2}+\sigma^{2} K_{t_{1}}-d \omega_{p}^{2}}{-\sigma^{2}+\sigma^{2} K_{t_{1}}+K_{t_{1}} K_{t_{2}} \sigma^{2}} \\
= & \frac{\sigma^{2}\left(-1+K_{t_{1}}-\frac{d \omega_{p}^{2}}{\sigma^{2}}\right)}{\sigma^{2}\left(-1+K_{t_{1}}+K_{t_{2}} K_{t_{2}}\right)}
\end{aligned}
$$

This can be further simplified to:

$$
e_{\text {proposed }_{(\text {steady-state) }}}=\frac{d}{K_{t_{2}} K_{t_{1}}}
$$

From (18), it is clear that zero steady-state error is almost achieved as $d<<K_{t_{2}} K_{t_{1}}$. With this analysis further supporting the claim that the proposed control scheme delivers superior positioning performance, full closed-loop experimental tests were carried out in both the frequency- and time-domain. The results and relevant discussion is presented in the following section.

\section{Results and discussion}

Figure 5 presents the frequency responses for the open-loop system (red), the traditionally controlled closed-loop (blue) and the proposed closed-loop controlled system (black). The plots suggest that the overall bandwidth of the proposed closed-loop scheme is somewhat lower than that of the traditional control scheme. To a certain extent, this is in line with the error dynamics plot presented in Figure 4. The time-domain plots for the traditional versus the proposed triangular 
trajectory tracking will clearly show that this slight loss of bandwidth is the cost paid for a significant improvement in tracking performance.
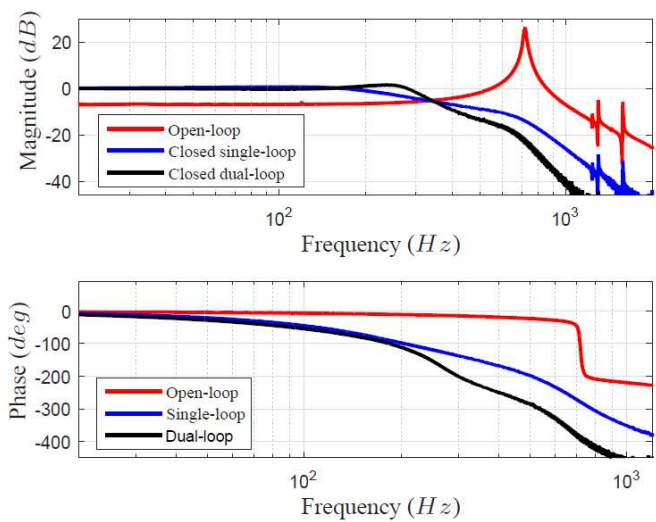

Figure 5. Frequency response of the system measured experimentally for the x-axis in open-loop versus closed-loop.

In order to test the tracking performance of the proposed method, a triangle signal of $30 \mathrm{~Hz}$ is selected as the reference. The closed-loop traces and errors for one period of the steady-state response are plotted in Figure 6 (a) and (b) for both cases.
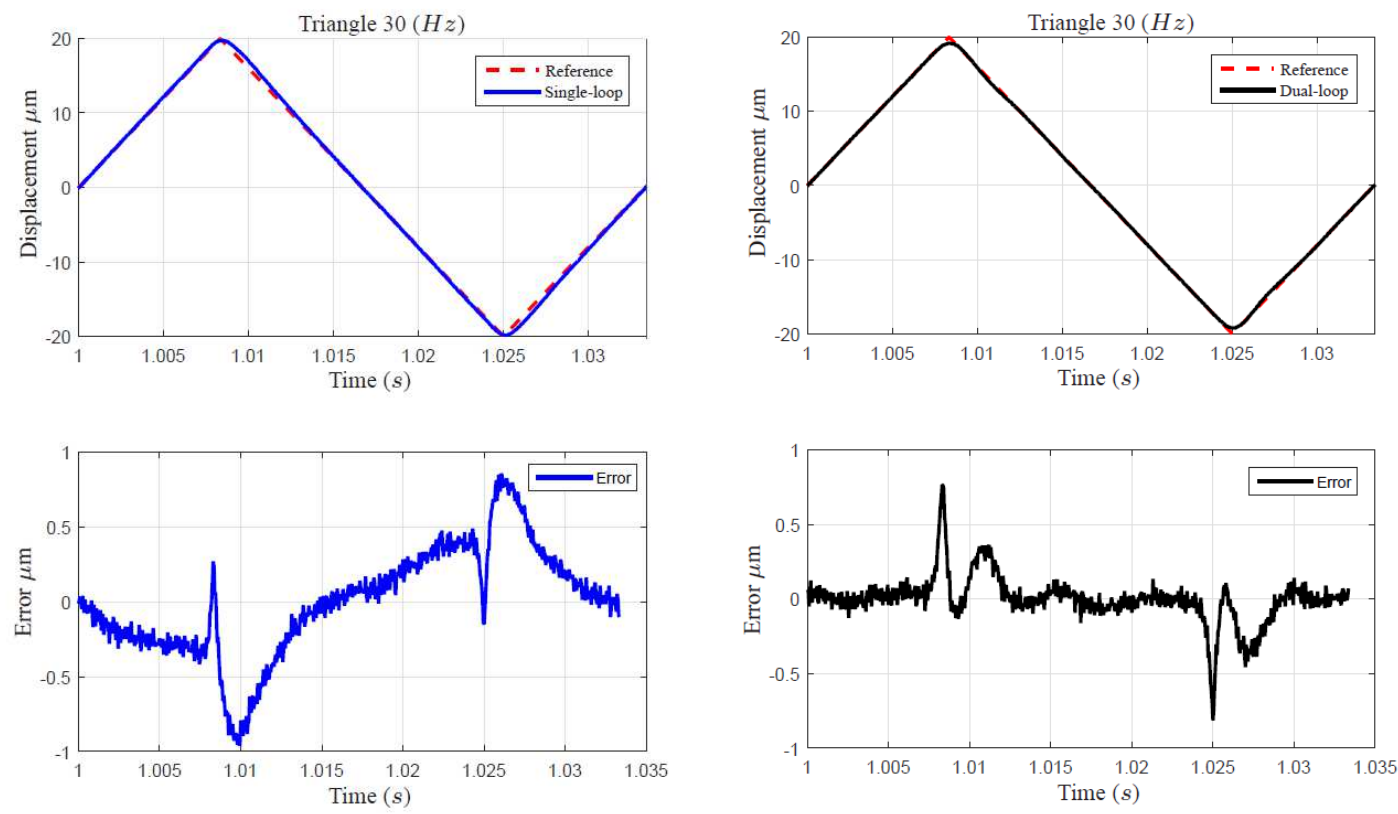

(a)

(b)

Figure 6. Comparison of the tracking performances: (a) the traditional single-loop with IRC; (b) the proposed multi-loop with IRC.

The results are then compared in order to evaluate the tracking performance, noting that it is desirable that the output of the system should track the input without generating any error. It is emphasised that the operating areas in the raster scan do not involve the corners of the triangle waveform (due to their high-frequency content) and they can be neglected. Figure 6 (b) shows that significant positioning errors occur along the entire trajectory in the traditional single-loop tracking feedback scheme.

The proposed dual-loop tracking strategy, on the other hand delivers a far superior tracking performance along the entire trajectory. A clear quantification of the errors for various frequencies tested is presented in Table 1. The maximum error 
and the root means square error (RMS) for triangular trajectories with different fundamental frequencies are compared for the traditional and the proposed method.

Table 1: Tabulates the maximum error and root mean square position error (RMS)

\begin{tabular}{|c|c|c|}
\hline Frequency $(\mathrm{Hz})$ & Maximum Error $(\mu \mathrm{m})$ & RMS Error $(\mu \mathrm{m})$ \\
\hline \multicolumn{3}{|c|}{ Single-loop } \\
\hline 10 & 0.4820 & 0.1248 \\
\hline 20 & 0.7787 & 0.2562 \\
\hline 30 & 0.8575 & 0.3645 \\
\hline \multicolumn{3}{|c|}{ Dual-loop } \\
\hline 10 & 0.2951 & 0.0668 \\
\hline 20 & 0.5749 & 0.0959 \\
\hline 30 & 0.7658 & 0.1571 \\
\hline
\end{tabular}

It is clear that the dual-loop scheme results in reduced tracking errors, both in the maximum and the RMS sense.

\subsection{Robustness}

Robustness is a measure of the insensitivity of the system performance to parameter changes, Das et al. (2015). In order for the closed-loop to be unresponsive to uncertainties, a reasonable stability margin is needed to cope with parameter changes. Nanopositioning platforms are usually susceptible to variations in resonance frequencies, Aphale et al. (2008). Different payload masses are applied to nanopositioning platforms, resulting in changing the resonance frequencies of the structure. Therefore, the robustness of the proposed control strategy was tested experimentally for a $15 \%$ reduction in resonant frequency. Figure 7 presents the frequency- and time-domain results for both the control schemes. It is clear that the proposed control scheme maintains superior tracking performance even under a change in resonant frequency of the axis.
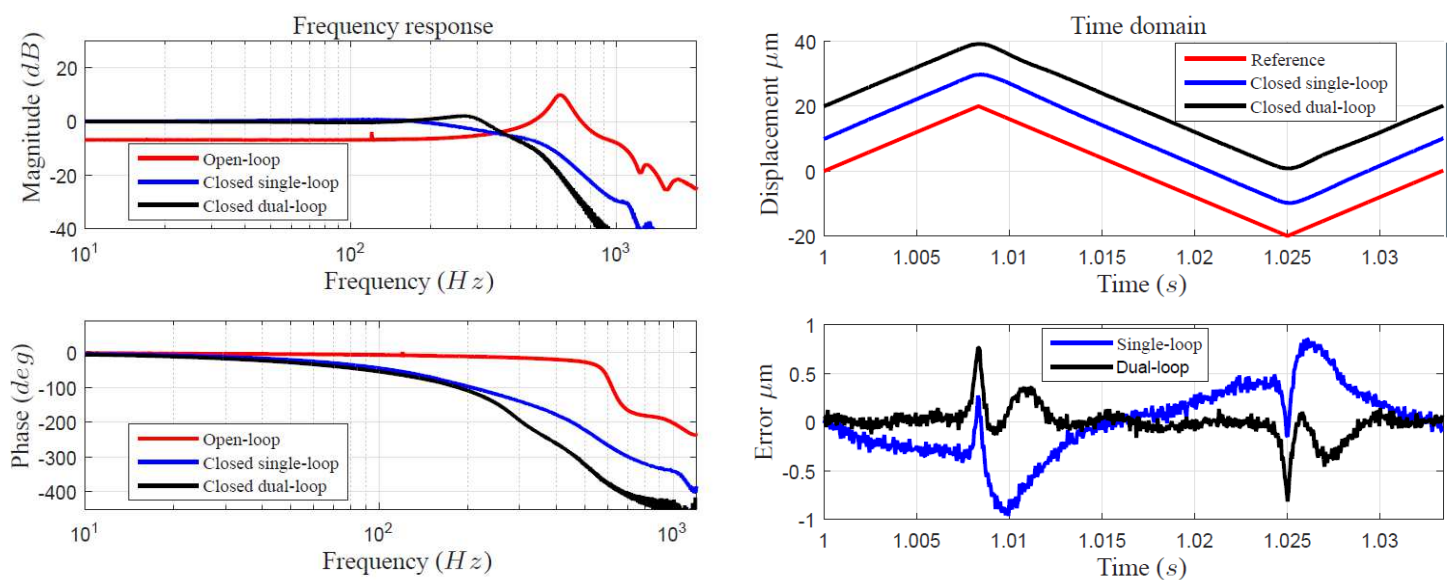

(a)

(b)

Figure 7. (a) Frequency response for the open-loop versus the closed-loop (single- and dual-loop) with a reduction in the resonant frequency of $15 \%$, from (716 to 613) $\mathrm{Hz}$ (mimicking loading); (b) a comparison of the closed-loop tracking performance for a $30 \mathrm{~Hz}$ triangle wave. 
The disturbance rejection profile of the proposed method is defined as in the equation below:

$$
\frac{Y(s)}{N(s)}=\frac{G(s)\left(1-C_{d}(s) d\right)}{1-C_{d}(s) d+G(s) C_{d}(s)+G(s) C_{t_{1}}(s) C_{d}(s)+G(s) C_{t_{2}}(s) C_{t_{2}}(s) C_{d}(s)}
$$

where $N(s)$ is a noise. Significant disturbance rejection is provided at frequencies lower than the resonant frequency. Similar to the traditional method, the disturbance rejection capability of the proposed control scheme is minimal around the resonance. The key benefit of the proposed dual-loop scheme lies therefore, in a delivering a substantially superior tracking performance, over a wide bandwidth, in a robust fashion.

\section{Conclusion}

In this article a new dual-loop tracking control strategy is proposed to further improve the positioning performance of a nanopositioner axis. An additional integral tracking controller is employed in conjunction with the traditional damping and tracking scheme in dual-loop feedback arrangement. The controller design procedures are simple and equations for controller parameter selection are derived. The experimental results confirm the feasibility and effectiveness of the proposed control strategy. Error analysis is presented to quantify this improvement. It is shown via experimental results that the proposed method produces a much smaller tracking error of triangular signals in the usable region of the raster scan. It is concluded that the proposed dual-loop control strategy can perform satisfactorily in nanopositioning applications required to scan ramp-like signals. Robustness of the proposed controller is examined in different loading conditions with no significant degradation of the tracking performance being observed.

\section{References}

Aphale, S. S., Devasia, S. \& Moheimani, S. O. R. (2008), 'High-bandwidth control of a piezoelectric nanopositioning stage in the presence of plant uncertainties', Nanotechnology 19(12), 125503.

Barrett, R. \& Quate, C. (1991), 'Optical scan-correction system applied to atomic force microscopy', Review of Scientific Instruments 62(6), 1393-1399.

Bhikkaji, B., Ratnam, M. \& Moheimani, S. O. R. (2007), 'Pvpf control of piezoelectric tube scanners', Sensors and Actuators A: Physical 135(2), 700-712.

Binnig, G., Quate, C. F. \& Gerber, C. (1986), ‘Atomic force microscope’, Physical Review Letters 56(9), 930.

Das, S. K., Pota, H. R. \& Petersen, I. R. (2015), ‘A mimo double resonant controller design for nanopositioners', IEEE Transactions on Nanotechnology 14(2), 224-237.

Devasia, S., Eleftheriou, E. \& Moheimani, S. O. R. (2007), ‘A survey of control issues in nanopositioning', IEEE Transactions on Control Systems Technology 15(5), 802-823.

Fanson, J. \& Caughey, T. K. (1990), 'Positive position feedback control for large space structures', AIAA Journal 28(4), 717-724.

Fleming, A. J. \& Leang, K. K. (2014), Feedback Control, Design, Modeling and Control of Nanopositioning Systems, Springer, pp. 175-219.

Fleming, A. J. \& Wills, A. G. (2009), 'Optimal periodic trajectories for band-limited systems', IEEE Transactions on Control Systems Technology 17(3), 552-562.

Gao, P., Li, X., Zhao, Z., Ma, X., Pu, M., Wang, C. \& Luo, X. (2017), 'Pushing the plasmonic imaging nanolithography to nanomanufacturing', Optics Communications .

Gu, G.-Y., Zhu, L.-M., Su, C.-Y., Ding, H. \& Fatikow, S. (2016), 'Modeling and control of piezo-actuated nanopositioning stages: A survey', IEEE Transactions on Automation Science and Engineering 13(1), 313-332.

Hagood, N. W. \& von Flotow, A. (1991), 'Damping of structural vibrations with piezoelectric materials and passive electrical networks', Journal of Sound and Vibration 146(2), 243-268. 
Ju, B.-F., Bai, X., Chen, J. \& Ge, Y. (2014), 'Design of optimal fast scanning trajectory for the mechanical scanner of measurement instruments', Scanning 36(2), 185-193.

Lee, C. (2017), Long-Range Nano-Scanning Devices Based on Optical Sensing Technology, Advanced Mechatronics and MEMS Devices II, Springer, pp. 495-522.

Li, C. X., Ding, Y., Gu, G. Y. \& Zhu, L. M. (2017), 'Damping control of piezo-actuated nanopositioning stages with recursive delayed position feedback', IEEE/ASME Transactions on Mechatronics 22(2), 855-864. ID: 1.

Mazlan, A. Z. A. \& Ripin, Z. M. (2017), 'The effective frequency range of an active suspended handle based on the saturation effects of a piezo stack actuator', Journal of Vibration and Control 23(5), 752-769.

McKelvey, T., Fleming, A. \& Moheimani, S. R. (2002), ‘Subspace-based system identification for an acoustic enclosure', Journal of Vibration and Acoustics 124(3), 414-419.

Namavar, M., Fleming, A. J., Aleyaasin, M., Nakkeeran, K. \& Aphale, S. S. (2014), 'An analytical approach to integral resonant control of second-order systems', IEEE/ASME Transactions on Mechatronics 19(2), 651-659.

Orszulik, R. R. \& Shan, J. (2017), 'Output feedback integral control of piezoelectric actuators considering hysteresis', Precision Engineering 47, 90-96.

Pota, H., Moheimani, S. O. R. \& Smith, M. (2002), 'Resonant controllers for smart structures', Smart Materials and Structures 11(1), 1 .

Rana, M., Pota, H. R. \& Petersen, I. R. (2018), 'A survey of methods used to control piezoelectric tube scanners in highâŁłspeed afm imaging', Asian Journal of Control .

Russell, D., San-Millan, A., Feliu, V. \& Aphale, S. S. (2016), 'Butterworth pattern-based simultaneous damping and tracking controller designs for nanopositioning systems. front', Mech.Eng 2(2).

San-Millan, A., Feliu-Batlle, V. \& Aphale, S. S. (2017), 'Fractional order implementation of integral resonant control - a nanopositioning application'. ID: 271436.

Sarella, A., Torti, A., Donolato, M., Pancaldi, M. \& Vavassori, P. (2014), 'Two-dimensional programmable manipulation of magnetic nanoparticles on-chip', Advanced Materials 26(15), 2384-2390.

Sebastian, A., Pantazi, A., Pozidis, H. \& Eleftheriou, E. (2008), 'Nanopositioning for probe-based data storage [applications of control]', IEEE Control Systems 28(4).

Sebastian, A. \& Salapaka, S. M. (2005), 'Design methodologies for robust nano-positioning', IEEE Transactions on Control Systems Technology 13(6), 868-876.

Soto, M. G. \& Adeli, H. (2017), 'Recent advances in control algorithms for smart structures and machines', Expert Systems 34(2).

Teo, Y. R., Russell, D., Aphale, S. S. \& Fleming, A. J. (2014), 'Optimal integral force feedback and structured pi tracking control: Application for objective lens positioner', Mechatronics 24(6), 701-711.

Wang, F.-C., Chen, L.-S., Tsai, Y.-C., Hsieh, C.-H. \& Yen, J.-Y. (2014), 'Robust loop-shaping control for a nano-positioning stage', Journal of Vibration and Control 20(6), 885-900.

Yong, Y. K., Bazaei, A. \& Moheimani, S. O. R. (2014), 'Video-rate lissajous-scan atomic force microscopy', IEEE Transactions on Nanotechnology 13(1), 85-93.

Zhu, Z., To, S., Ehmann, K. F. \& Zhou, X. (2017), 'Design, analysis, and realization of a novel piezoelectrically actuated rotary spatial vibration system for micro-/nano-machining', IEEE/ASME Transactions on Mechatronics 22(3), 1227 - 1237. 